\title{
Evidence for channel conduction in low misorientation angle [001] tilt $\mathrm{YBa}_{2} \mathrm{Cu}_{3} \mathrm{O}_{7-x}$ bicrystal films
}

\author{
N. F. Heinig, ${ }^{\text {a) }}$ R. D. Redwing, I Fei Tsu, A. Gurevich, J. E. Nordman, S. E. Babcock, \\ and D. C. Larbalestier \\ Applied Superconductivity Center, University of Wisconsin, Madison, Wisconsin 53706
}

(Received 2 October 1995; accepted for publication 17 May 1996)

\begin{abstract}
Extended voltage-current $(V-I)$ characteristics of [001] tilt $\mathrm{YBa}_{2} \mathrm{Cu}_{3} \mathrm{O}_{7-x}$ bicrystal films having misorientation angles, $\theta$, of $3^{\circ}$ to $20^{\circ}$ showed a substantial and progressive change with $\theta$, when measured in large fields. Transmission electron microscopy of the $10^{\circ}$ bicrystal showed the grain boundary to contain edge dislocations separated by channels of relatively undisturbed lattice. A large drop in the intergrain irreversibility field, $H^{*}$, occurred between $10^{\circ}$ and $15^{\circ}$, and the characteristics became qualitatively different by $20^{\circ}$. Both the microscopy and the electromagnetic data support a heterogeneous description of the grain boundary, consisting of strongly coupled channels that close at a misorientation angle of around $15^{\circ}$. (c) 1996 American Institute of Physics. [S0003-6951(96)03630-3]
\end{abstract}

It is well established that the critical current density, $J_{c}$, of [001] tilt thin film bicrystals of high temperature superconductors is strongly dependent on the misorientation angle, $\theta$, of the grain boundary (GB).$^{1-4}$ Dimos et al. ${ }^{1}$ proposed that the cores of primary grain boundary dislocations (PGBD) subdivide low angle grain boundaries. Chisholm and Pennycook ${ }^{5}$ additionally proposed that the strain fields of the PGBDs extend the effective size of the dislocation cores. Both models postulate that the supercurrent is restricted to channels of good lattice between the PGBDs. The effective size of the PGBD cores should then determine the magnitude of $J_{b} / J_{c}$ (the ratio of the inter- to intragranular critical current density), and the critical misorientation angle at which strong coupling properties disappear. Measurements on different kinds of samples suggest that this critical angle can vary over a wide angular range of $5^{\circ}-20^{\circ}{ }^{1,4,6,7}$ Since this range covers a PGBD separation of $4.5-0.6 \mathrm{~nm}$, a more explicit test of the above models is highly desirable. ${ }^{8}$ This issue has regained importance because of renewed activity on making conductors with a strong biaxial texture from $\mathrm{YBa}_{2} \mathrm{Cu}_{3} \mathrm{O}_{7-x}$ (YBCO), using an ion beam assisted deposition process. ${ }^{9-11}$ Since such conductors would be useful for the production of strong fields, provided that the spread in the $a / b$ grain axis misalignment is controlled, knowledge of the extended high field voltage current $(V-I)$ characteristics of YBCO bicrystals of varying misorientations is of both fundamental and applied interest.

This letter describes the first systematic measurements of the $V-I$ curves of [001] tilt YBCO bicrystal thin films with misorientation angles in the low-to-high angle crossover regime of $3^{\circ}-20^{\circ}$ in large magnetic fields. Prior measurements of single crystal thin film ${ }^{12,13}$ and bulk ${ }^{14}$ high temperature superconductors have established that the irreversibility field, $H^{*}$, above which $J_{c}$ tends to zero, can be experimentally defined by the change in sign of the curvature of the electric field-current density, $E-J$, characteristic plotted on a double logarithmic scale. In single crystal YBCO films, this transition occurs near $4 T$ at $77 \mathrm{~K}^{12}$ To test the channel models

${ }^{\text {a)} E l e c t r o n i c ~ m a i l: ~ h e i n i g @ c a e . w i s c . e d u ~}$ described above, we compared these well-established intragrain properties to the intergrain properties, reasoning that $H^{*}$ would be severely depressed if the boundary consists of overlapping PGBD cores or strain fields. As long as open channels remain, vortices at the GB can interact strongly with vortices in the grains, leaving $H^{*}$ comparable to single crystal values. Thus, determination of $H^{*}$ as a function of $\theta$ provides an explicit measure of the critical misorientation angle between strong and weak coupling. Previous studies of thin-film bicrystals in high fields ${ }^{15-17}$ have not addressed this point, nor the specific nature of the electromagnetic coupling in bicrystals with $\theta$ lying in the transition range.

$C$-axis oriented YBCO films of 100-250 nm thickness were made by pulsed laser deposition onto [001] symmetric tilt $\mathrm{SrTiO}_{3}$ bicrystal substrates. ${ }^{18}$ The films were patterned and ion beam etched to make bridges of various width between 5 and $100 \mu \mathrm{m}$. The voltage probes were $500 \mu \mathrm{m}$ apart across the grains and $100 \mu \mathrm{m}$ apart across the GB. The film transition temperatures were $87-89 \mathrm{~K}$ and the intragranular zero field $J_{c}(77 \mathrm{~K})$ values were $1-5 \mathrm{MA} / \mathrm{cm}^{2}$, indicating good film quality.

Zero field measurements were made in a mu-metal shielded dewar in a screened room, and high field measurements in a $15 \mathrm{~T}$ superconducting magnet. All measurements were made with the magnetic field, $H$, perpendicular to the film plane (i.e., parallel to the $c$ axis). High sensitivity $V-I$ curves were taken with a nanovoltmeter and a precision current supply over almost five decades of voltage $(5 \mathrm{nV}-100$ $\mu \mathrm{V})$, and four decades of current (10 nA-100 mA). The data are presented in voltage-current density $(V-J)$ form, because the electric field across the intergrain link is clearly nonuniform in the vicinity of the grain boundary.

Figure 1 shows extended $V-J$ curves at $77 \mathrm{~K}$ in fields up to $8 \mathrm{~T}$ for an intragrain link, that is $\theta=0^{\circ}$, and for intergrain links across $5^{\circ}, 10^{\circ}, 15^{\circ}$, and $20^{\circ}$ [001] tilt boundaries. The curve sets show a distinctive and progressive change as $\theta$ increases. The intragrain and the $3^{\circ}$ intergrain $V-J$ curves are power law-like and have the general characteristics observed by Koch et al. ${ }^{12}$ on single crystal YBCO thin films. At and above $5^{\circ}$, the $V-J$ curves exhibit a marked change. A 

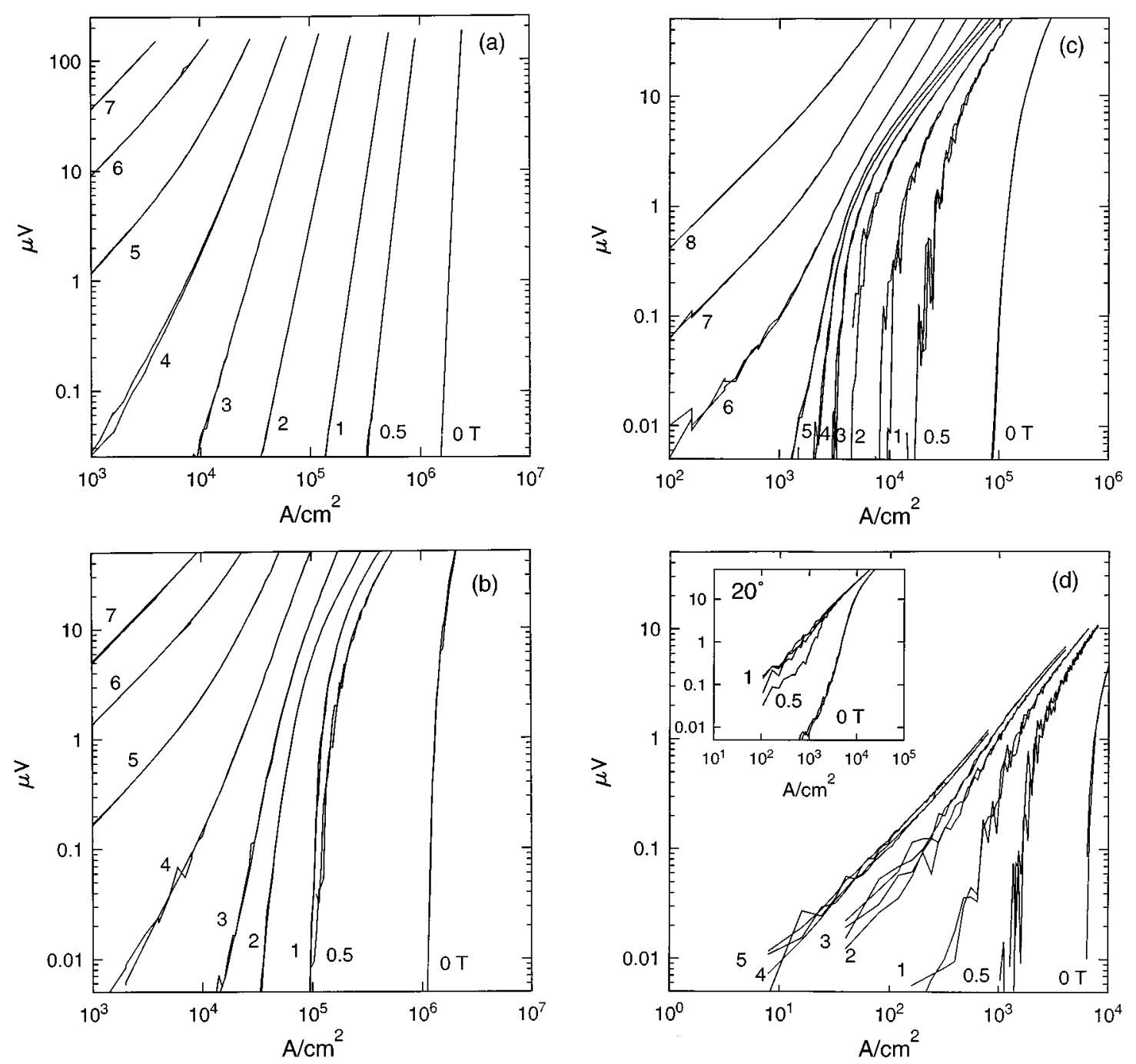

FIG. 1. Current density vs voltage in high magnetic fields at $77 \mathrm{~K}$. (a) Intragrain link, (b) $5^{\circ}$ link, (c) $10^{\circ}$ link, and (d) $15^{\circ}$ link, with the inset showing a $20^{\circ}$ link.

strong bunching of the lower field curves indicates that there is a weak-linked component to the intragrain $J_{c}$. There is also a tendency towards ohmic behavior at higher $V$, particularly for the $15^{\circ}$ and $20^{\circ}$ bicrystals, showing the effect of flux flow in the increasingly resistive boundaries. Another feature is that the intergrain voltage noise increases with increasing misorientation angle. It appears likely that the noise is due to fluxon motion in Josephson-coupled components of the grain boundary.

In order to extract information about the strongly coupled components of the GBs, the data in Fig. 1 were analyzed by fitting the low voltage portion $(<1 \mu \mathrm{V})$ of the $\log V / \log I$ data to a quadratic equation. The sign of the quadratic term changes at the irreversibility field, $H^{*}$. The higher voltage portions in the intergrain links were not included in the fit, since they correspond to flux flow, consistent with the high electric field values in the vicinity of the grain boundary. The intragrain $H^{*}(77 \mathrm{~K})$ ranged from 2.5 to $4 \mathrm{~T}$, with an uncertainty of $\sim 0.5 \mathrm{~T}$. For the $3^{\circ}$ and $5^{\circ}$ bicrystals, $H^{*}(77 \mathrm{~K})$ was $3 \mathrm{~T}$. For the $7^{\circ}, H^{*}$ was $3.5 \mathrm{~T}$, and for the $10^{\circ}, H^{*}$ was $5.5 \mathrm{~T}$. This increase in $H^{*}$ with increasing $\theta$ may be explained by stronger flux pinning by the increasingly closely spaced PGBDs. However, beyond $10^{\circ}$,
$H^{*}\left(77 \mathrm{~K}\right.$ ) dropped to $0.75 \mathrm{~T}$ for the $15^{\circ} \mathrm{GB}$, and by $20^{\circ}$, no low voltage negative curvature was seen, indicating the whole GB was weakly coupled. We take this large depression in $H^{*}(77 \mathrm{~K})$ beyond $10^{\circ}$ to be a clear signature that the strongly coupled channels are extinguished near $15^{\circ}$.

Figure 2 is a Fourier-filtered high resolution transmission electron microscopy image of one $10^{\circ}$ bicrystal. It shows the [001] projection of both crystals. The two $c$ axes

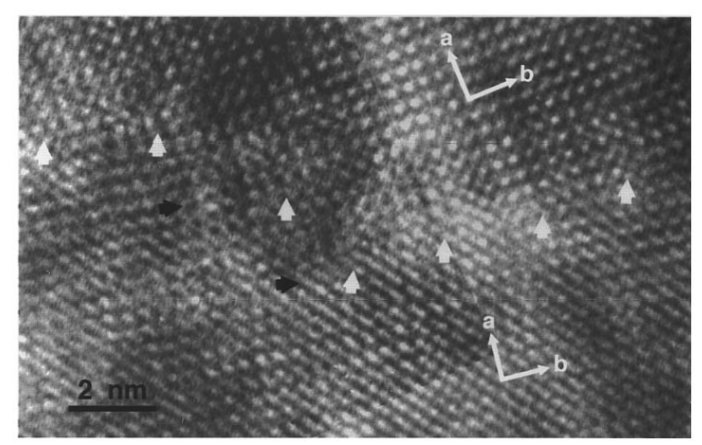

FIG. 2. High resolution transmission electron image of a $10^{\circ}$ thin-film $\mathrm{YBa}_{2} \mathrm{Cu}_{3} \mathrm{O}_{7-x}$ grain boundary. 


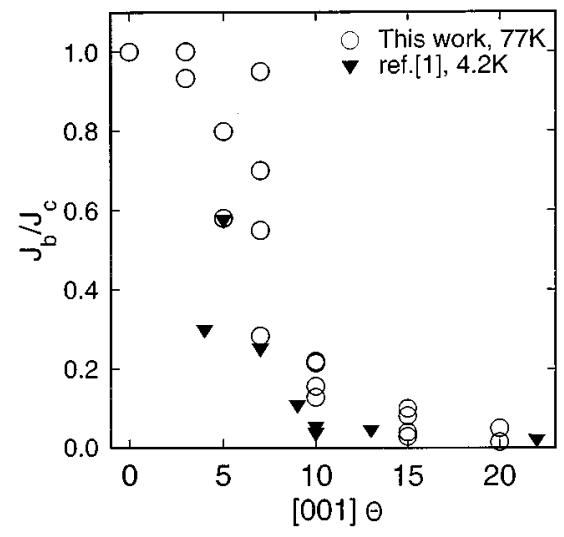

FIG. 3. The ratio of the zero magnetic field intergrain and intragrain critical current density, $J_{b} / J_{c}$ vs [001] misorientation angle. The open circles are from this work, taken at $77 \mathrm{~K}$, and the closed triangles are from Ref. 1, taken at $4.2 \mathrm{~K}$.

lie within $1^{\circ}$ of each other. PGBDs in the boundary appear as terminating lattice planes in the image. Two kinds of PGBDs are observed in this section of the boundary, those with Burgers vectors, $\mathbf{b}=[100]$ (white arrows), and those with $\mathbf{b}=[010]$ (dark arrows). In the right half of Fig. 2, the boundary is a symmetric [001] tilt boundary with the ideal Frank dislocation spacing ${ }^{19}$ of $2.2 \mathrm{~nm}$. If one approximates the effective dislocation core diameter as $2|\mathbf{b}|$, the width of the channels between the dislocation cores is $1.4 \mathrm{~nm}$. This value is close to the coherence length, $\xi$, indicating that both the electromagnetic and microstructural data support a filamentary channel model. Consistent with earlier studies of "wavy" boundaries in thin-film YBCO bicrystals, ${ }^{20-22}$ the boundary deviates from this ideal inclination in the left part of Fig. 2.

In earlier studies, ${ }^{1-5}$ the transition from strong to weak coupling was evaluated by plotting zero field properties, $J_{b}$ or $J_{b} / J_{c}$ vs $\theta$. In Fig. 3, zero field $J_{b} / J_{c}$ for our samples is compared to earlier work ${ }^{1}$ on electron beam evaporated samples. $J_{b} / J_{c}$ for the laser ablated bicrystals is not very dependent on $\theta$ up to about $7^{\circ}$, and then shows the exponential decrease in $J_{b}$ with $\theta$ seen by other groups. ${ }^{2}$ Film-to-film variations in the intragrain $J_{c}$ contribute to scatter in the data, and, taken together with the differences in $J_{b} / J_{c}$ between the evaporated films, ${ }^{1}$ and the laser ablated films, indicate that the strong-to-weak coupling transition is sample, and perhaps processing, dependent. In any case, the smooth exponential decrease in $J_{b} / J_{c}$ with misorientation angle makes the closure of any strongly coupled channels less obvious in the zero field data, than when it is observed by the high field $H^{*}$ transitions of Fig. 1. Additional discussions of this way of analyzing the channel properties of low angle bicrystals is presented in a recent study of an $8^{\circ}$ [001] tilt $\mathrm{Bi}_{2} \mathrm{Sr}_{2} \mathrm{CaCu}_{2} \mathrm{O}_{x}$ bulk bicrystal by Wang et al. ${ }^{23}$

In summary, a systematic study of the $V-I$ curves of [001] tilt $\mathrm{YBa}_{2} \mathrm{Cu}_{3} \mathrm{O}_{x}$ bicrystal films in large magnetic fields shows a gradual evolution of their $V-I$ curves in the misorientation angle range of $3^{\circ}-20^{\circ}$. The observed changes support models that postulate that strongly coupled segments of the GB lie between primary grain boundary dislocations. The grain boundaries in these thin-film bicrystals appear to lose their strongly coupled channels due to the overlap of dislocation cores or strain fields at a misorientation angle close to $15^{\circ}$.

This work has been supported by the NSF (Materials Research Group Program) under Contract No. DMR9214707. Experimental assistance from Cai Xue Yu is gratefully acknowledged.

${ }^{1}$ D. Dimos, P. Chaudhari, and J. Mannhart, Phys. Rev. B 41, 4038 (1990); D. Dimos, P. Chaudhari, J. Mannhart, and F. K. LeGoues, Phys. Rev. Lett. 61, 219 (1988).

${ }^{2}$ R. Gross, in Interfaces in Superconducting Systems, edited by S. L. Shindé and D. Rudman (Springer, New York, 1992), p. 176.

${ }^{3}$ T. Amrein, L. Schultz, B. Kabius, and K. Urban, Phys. Rev. B 51, 6792 (1995)

${ }^{4}$ Z. G. Ivanov, P. Å. Nilsson, D. Winkler, J. A. Alarco, T. Claeson, E. A. Stepantzov, and A. Ya. Tzalenchuk, Appl. Phys. Lett. 59, 3030 (1991).

${ }^{5}$ M. F. Chisholm and S. J. Pennycook, Nature 351, 47 (1991).

${ }^{6}$ M. B. Field, X. Y. Cai, S. E. Babcock, and D. C. Larbalestier, IEEE Trans. Supercond. 3, 1479 (1993).

${ }^{7}$ D. W. A. Willén and K. Salama, Physica C 201, 311 (1992).

${ }^{8}$ S. E. Babcock and J. L. Vargas, Annu. Rev. Mater. Sci. 25, 193 (1995).

${ }^{9}$ Y. Iijima, N. Tanabe, O. Kohno, and Y. Ikeno, Appl. Phys. Lett. 60, 769 (1992).

${ }^{10}$ R. P. Reade, P. Berdahl, R. E. Russo, and S. M. Garrison, Appl. Phys. Lett. 61, 2231 (1992).

${ }^{11}$ X. D. Wu et al.,Appl. Phys. Lett. 65, 1961 (1994).

${ }^{12}$ R. H. Koch, V. Foglietti, W. J. Gallagher, G. Koren, A. Gupta, and M. P. A. Fisher, Phys. Rev. Lett. 63, 1511 (1989).

${ }^{13}$ H. Yamasaki, K. Endo, S. Kosaka, M. Umeda, S. Yoshida, and K. Kajimura, Cryogenics 35, 263 (1995).

${ }^{14}$ Q. Li, H. J. Weismann, M. Suenaga, L. Motowidlo, and P. Haldar, Phys. Rev. B 51, 701 (1995).

${ }^{15}$ M. Däumling, E. Sarnelli, P. Chaudhari, A. Gupta, and J. Lacey, Appl. Phys. Lett. 61, 1355 (1992).

${ }^{16}$ E. Sarnelli, P. Chaudhari, and J. Lacey, Appl. Phys. Lett. 62, 777 (1993).

${ }^{17}$ O. M. Froehlich, H. Schulze, A. Beck, B. Mayer, L. Alff, R. Gross, and R. P. Huebener, Appl. Phys. Lett. 66, 2298 (1995).

${ }^{18}$ Purchased from the Shinkosha Co, 4-8-8 Ginza, Chuo-Ku, Tokyo 104, Japan.

${ }^{19}$ e.g., J. P. Hirth and J. Lothe, in Theory of Dislocations (Wiley, New York, 1982).

${ }^{20}$ J. A. Alarco, E. Olsson, Z. G. Ivanov, P. Å. Nilsson, D. Winkler, E. A. Stepantsov, and A. Y. Tzalenchuk, Ultramicroscopy 51, 239 (1993).

${ }^{21}$ C. Traeholt, J. G. Wen, H. W. Zandbergen, Y. Shen, and J. W. M. Hilgenkamp, Physica C 230, 425 (1994).

${ }^{22}$ D. J. Miller, T. A. Roberts, J. H. Kang, J. Talvacchio, D. B. Buchholz, and R. P. H. Chang, Appl. Phys. Lett. 66, 2561 (1995).

${ }^{23}$ J. L. Wang, I. F. Tsu, X. Y. Cai, R. J. Kelley, M. D. Vaudin, S. E. Babcock, and D. C. Larbalestier, J. Mater. Res. 11, 868 (1996). 